BoL. Soc. Bot. MÉxico 30:97-112, 13 figs., 2 mapas (1969)

\title{
INFLUENCIA DE LAS AVES EN LA VEGETACION DE LA LAGUNA DEL MAJAHUAL EN LOS TUXTLAS, VER.
}

\section{AGRADECIMIENTOS}

Mario Sousa S.*

Sin la estrecha colaboración recibida del Dr. Allan R. Phillips, la realización de este trabajo hubiera sido poco menos que imposible. Su ayuda comprendió tanto observaciones e identificaciones como revisión del texto.

Debemos agradecer al Dr. Daniel H. Janzen el habernos enviado información inédita de inestimable valor.

Al Dr. Arturo Gómez-Pompa sus adecuadas sugestiones.

A la Sra. Biól. Magdalena Peña de Sousa por sus excelentes dibujos, los cuales exponen en forma clara los objetivos del trabajo.

A la Sra. Margarita Peña que le imprimió a la redacción una mayor nitidez.

$\mathrm{Y}$ en los trabajos de campo a los señores Refugio Cedillo T. y Rafael Hernández.

\section{RESUMEN}

Se colectaron 17 especies vegetales en la Laguna del Majahual en Los Tuxtlas, Veracruz, con un marcado contraste entre el rojo de frutos, semillas, arilos, inflorescencias o ramas y el negro de frutos o semillas o el blanco de arilos o valvas internas de frutos.

Como hipótesis de trabajo se propone a las aves como agentes que propiciaron la concentración de especies vegetales con las características convergentes ya citadas.

Se observaron y colectaron 11 especies de aves frugívoras de las cuales 2 son aves migratorias del NE y SE de los Estados Unidos y Canadá respectivamente, las que se alimentaban de 5 de las especies vegetales con rojos contrastantes.

Se hace un breve estudio sinecológico de la Laguna incluyendo datos físicos del medio ambiente, proponiéndose a los taludes oriental y occidental como refugios de aves en la época de los "nortes" que permite la "siembra" de especies vegetales que son su alimento en este período desfavorable.

\section{INTRODUCCION}

A principios de noviembre de 1967 realizamos un viaje de colecta botánica a la Laguna del Majahual en la región de Los Tuxtlas, Ver. (mapa 1). Nos llamó mucho la atención la abundancia de Swartzia guatemalensis, un arbusto de 1.5

\footnotetext{
* Departamento de Botánica del Instituto de Biología, UNAM.
} 
a $2.5 \mathrm{~m}$. de alto. Era bastante notorio por sus frutos, que son anaranjados, dehiscentes con una sola semilla negra que pende y de su extremo basal a su parte media está cubierta por un arilo blanco (fig. 1).

En la misma localidad y fecha colectamos a Connarus schultesii (fig. 2), Guarea glabra (fig. 3), Paullinia pinnata (fig. 4) y Pithecellobium arboreum (fig. 5). Todas estas especies tienen frutos rojos, semillas negras (excepto Guarea que es roja) y arilos blancos o amarillos (Connarus), excepto Guarea y Pithecellobium.

Regresamos los últimos días de noviembre con el propósito de realizar una colecta más detallada, encontrando además de las especies referidas:

Mortoniodentron sp. (fig. 6), Trichilia tomentosa (fig. 7) y Trichilia breviflora (fig. 8) con cápsulas cuyas semillas estaban cubiertas con arilos rojos.

Cymbopetálum baillonii (fig. 9) y Tabernaemontana alba con folículos rojizos y arilos rojos.

Casearia nitida: (fig. 10) cápsula con una semilla con arilo anaranjado.

Virola multiflora (fig. 11) : cápsula con una sola semilla con arilo anaranjado laciniado.

Chamaedorea tepejilote (fig. 12) con pedúnculos anaranjados y frutos negros.

Bursera simaruba (fig. 13): frutos pardos y ramas rojizas.

Psychotria uliginosa (fig. 14): frutos rojos.

Smilax domingensis (fig. 15): frutos rojos.

Malmea depresa (fig. 16): frutos compuestos rojos.

Nos preguntamos, ¿por qué esta convergencia de rojo en frutos, semillas, arilos, inflorescencias o ramas, generalmente contrastada con negro de frutos 0 semillas, o blanco de arilos o caras internas de las valvas de los frutos? Además, ¿por qué son abundantes?, ¿por qué en la Laguna del Majahual? y ¿por qué en esta fecha?

En los frutos dehiscentes (cápsulas o folículos) las semillas persisten en el fruto y en ocasiones el fruto cae de la planta antes que las semillas se desprendan de él, como llega a suceder en Pithecellobium arboreum. Los frutos carnosos generalmente están arreglados en inflorescencias o son frutos compuestos (como en Malmea).

Como ha hecho ver Corner (1949) en las selvas tropicales actuales, de los árboles y bejucos quizá el $5 \%$ tienen semillas ariladas o cubiertas pulposas. De lo que ha sido estudiado de la flora leñosa de la Selva Alta Perennifolia de Los Tuxtlas, se infiere que son pocas las plantas con semillas ariladas y de esas pocas casi todas están representadas, al menos a nivel genérico, en la Laguna del Majahual, faltando: Maytenus belizensis, Sloanea sp. y especies de géreros ya representados como: Mortoniodendron guatemalensis, Paullinia tomentosa y Virola 
guatemalensis. Muchas de ellas quizá habitaron en los taludes de la Laguna, pero debido a la alteración que abarca un 50\% de los taludes de la laguna cráter, posiblemente fueron eliminadas. Sin embargo Mortoniodendron sp. (muy posiblemente una especie nueva), Virola multiflora (especie nueva para la flora de México) y Connaruss shultesii (segunda colecta después del Tipo) dentro de la región de Los Tuxtlas sólo se les ha encontrado en dicha Laguná.

De las 16 especies encontradas, 9 son árboles, 3 son bejucos y 4 son arbustos. Los árboles miden de 6 a $22 \mathrm{~m}$. de alto, los bejucos fructifican en el dosel de la selva y los arbustos tienden a crecer en la orilla de la Laguna.

$\mathrm{Si}$ pensamos que el responsable de esta convergencia en tiempo y espacio es algún tipo de animal, ya que, los arilos y testas pulposas son ricas en aceites, carotenoides y otras sustancias complejas (Corner, op. cit.) lo que les proporciona un valioso alimento, debemos incluir a aquellos animales a los que de alguna forma les llaman la atención estas características pomológicas.

En las orillas de la Laguna del Majahual se observó la presencia de dos especies de ardillas:

Sciurus hernandezii, la ardilla roja y

Sciurus deppei la ardilla negra, siendo la primera mucho más abundante que la segunda.

No se observaron primates debido a que los alrededores de la laguna están sumamente alterados, pero es muy posible que ni primates ni roedores sean los responsables de la convergencia carpológica, ya que ambos grupos destruyen a las semillas, o bien en el caso de los roedores su nicho es muy reducido y sus posibilidades dispersoras son mínimas.

Las aves y murciélagos frugívoros son otros organismos que podrían converger a estas plantas. Nos inclinamos por las aves a las que por su talla les es posible alimentarse de semillas de mayor tamaño. Su carencia en dientes evita la destrucción de las semillas (eliminando a los grupos granívoros y a los pericos y cotorras que dañan a los simientes). Por otro lado, el rojo es el color de las flores que fundamentalmente son polinizadas por las aves.

\section{RESULTADOS}

Con los antecedentes referidos se iniciaron las observaciones de aves frugívoras.*

Por medio de la observación directa o por el examen del contenido intesti-

* Se invitó al doctor Allan R. Phillips a un viaje de tres días a la laguna, más cinco días de observación realizada por el autor. Siendo ocho días un período muy corto de observación; estamos seguros que el número de aves frugívoras que visitan a la laguna debe ser mucho mayor al observado. 
nal de diversas aves se encontró que las siguientes se alimentan de Bursera simaruba:

\author{
Caryothraustes poliogaster \\ Myiozetetes similis \\ Pitangus sulphuratus \\ Psilorhinus mexicanus \\ Pteroglossus torquatus \\ Tityra semifasciata
}

Wetmore (1943) también encontró en Los Tuxtlas que Myiozetetes similis se alimenta de frutos del "palo mulato" (Bursera simaruba). Se observó que esta especie de ave llega en parvadas de 8 a 10 individuos a un solo árbol. Se cazó a un ejemplar y le fueron encontradas 5 semillas del referido árbol. En la prueba de germinación un 50\% respondió positivamente (se sembraron 4 semillas).

En el contenido intestinal de un ejemplar de Psilorhinus mexicanus ("pepe" papán o pea) encontramos 21 semillas de Bursera simaruba; de las 10 que se sembraron germinó un 50 por ciento.

Es interesante notar que el porcentaje de germinación de esta especie es menor al uno por ciento en condiciones de viveros forestales (Sarukhan, 1963).

Hay pocas dudas de que las aves jueguen un importante papel en el logro de una mayor amplitud ecológica, en la dispersión y distribución geográfica de Bursera simaruba.

Corner (op. cit.) ha hecho ver el estrecho mutualismo que existe entre los animales frugívoros y los frutos. Así dice que existe una gran presión animal para alimentarse de frutos inmaduros con una consecuente pérdida de semillas; sin embargo, los frutos se protegen por medio de: 1) el mimetismo de su verde con el de las hojas; 2) protección mecánica y 3) protección química debida a la impalatabilidad causada por la acidez. A este respecto D. H. Janzen (ined.) menciona que es particularmente notable cómo han sido observadas aves probando frutos inmaduros de Bursera simaruba, de las bajas altitudes de Centroamérica y rechazándolos de inmediato (protección química) o bien tratando inútilmente de arrancarlos inmaduros (protección mecánica).

Las siguientes aves se alimentan de Trichilia tomentosa:

Myiozetetes similis

Pipromorpha oleaginea

Tityra semifasciata

Trogon violaceus

Turdus grayi 
En el tracto digestivo de un ejemplar de Tityra semifasciata se colectaron 8 semillas de esta Trichilia (no fue hecha la prueba de germinación). En las demás especies de aves el dato se obtuvo por medio de la observación directa.

En el tracto digestivo del tucán Pteroglossus torquatus ("pico de canoa") se colectaron semillas de: Bursera simaruba, Paullinia pinnata, Psychotria chiapensis y una especie no identificada. Es interesante notar que este tucán vive en troncos huecos de árboles como se observó en un árbol de Coccoloba aff. humboldtii en la laguna.

En el volcán de San Martín Tuxtla, a 1200 m. de altitud, se colectó a una pareja de tucanes verdes (Aulacorhynchus prasinus), especie que vive en el bosque caducifolio. Su contenido intestinal lo formaban: Paullinia pinnata, Rubus sp. y Trichilia glabra. Es de notarse la semejanza en la dieta de ambas especies de tucanes, ya sean de la selva alta perennifolia o del bosque caducifolio.

En el tracto digestivo de un "pepe" (Psilorhinus mexicanus) se encontraron semillas de Bursera simaruba y Smilax domingensis y un escarabajo del género Xyloryctes, lo que nos da una idea de su dieta mixta.

Andrle (1967) encontró en el buche del pichón Columba speciosa fruto:s (rojos) de Pseudolmedia oxyphyllaria, en Los Tuxtlas a fines de abril de 1962.

Entre las aves migratorias del NE de los Estados Unidos y SE de Canadá, Dumetella carolinensis contenía semillas de Psychotria aff. uliginosa, e Icterus galbula, de Crotalaria sp.

Con la idea de observar a las aves que se alimentan de Swartzia guatemalensis se colectaron:

Arremonops rufivirgatus y

Dendroica magnolia, migratoria del norte. Ambas especies son pequeñas, y los ejemplares cazados se alimentahan de los insectos que visitan al arilo de Swartzia.

Pensamos que por el gran tamaño de las semillas de Connarus schultesii, Swartzia guatemalensis y Virola multiflora no deben pasar por todo el tracto digestivo de ave alguna, pero deben ser regurgitadas ya sin arilo por los tuca. nes; de hecho observamos a una pareja de Pteroglossus torquatus que regurgi. taban y excretaban a la vez en el vuelo. También se observó la presencia del tucán Ramphastos sulfuratus. Por el gran tamaño del pico de estas dos especies de tucanes es fácil que tomen las grandes semillas de las especies vegetales citadas y por medio de sus lenguas plumosas remuevan los arilos y eliminen las semillas. Hespenheide (1966) realizó experimentos en fringílidos (pinzones o gorriones) en cuanto al tamaño de su alimento y al tamaño y forma de sus picos, encontrando una estrecha correlación entre ambos, o sea los pinzones de 
pico grande, tienden a alimentarse con comida de mayor talla que la seleccionada por los pinzones de pico pequeño.

Sin embargo en los tucanes colectados, los frutos y semillas de su contenido intestinal no correspondían al tamaño y forma de sus picos, siendo en general frutos pequeños, lo que no descarta la posibilidad de correspondencia eventual entre el tamaño del fruto o semilla y el pico de estas aves, como ya se sugirió. Diamond y Terborgh (1967) hacen notar este hecho, señalando la heterogeneidad de grupos taxonómicos de aves, incluyendo tamaños y formas de picos, que se alimentan de manera sincrónica o no de una misma especie vegetal.

Janzen (op. cit.) hace una interesante observación en cuanto a "las especies con semillas grandes, suaves y de colores negros, pardos o moteados (o frutos indehiscentes que cubren semillas suaves) que son fácilmente destruidas por aves, las que a la vez las dispersan (numerosas Lauraceae, Annonaceae, Pithecellobium arboreum, pequeñas Palmae, etc.)" ... Continúa Janzen: "Estas plantas tienen color rojo o anaranjado en pedúnculos, brácteas, receptáculos, o tallos que aparentemente sirven de atracción a las aves..." y agrega "...Esto sugiere la siguiente hipótesis: si las semillas son comidas, son destruidas, pero si son tiradas las semilas no pueden ser encontradas de nuevo por el ave (u otro herbívoro) y así la dispersión de la semilla por sus depredadores es posible (mientras que un fruto o semilla roja serían más fáciles de relocalizar)."

Se debe agregar que en las aves de la selva primaria hay cierta territoria lidad vertical (Phillips, comunicación personal), lo que dificultaría aún más un intento en la húsqueda del ave por simientes en el suelo.

\section{Vegetación}

Los taludes oriental y occidental de la Laguna del Majahual sustentan a una selva mediana perennifolia y los taludes norte y sur a una selva caducifolia, a finales de la época de los "nortes". A pesar de las diferencias fisonómicas de los taludes la asociación de 18 a $22 \mathrm{~m}$. de alto, tiene como dominantes a:

Diospyros digyna Jacq.

Bursera simaruba (L.) Sarg.

Coccoloba aff. humboldtii Meisner

En la orilla de la Laguna Diospyros digyna Jacq. comparte la dominancia con Pachira aquatica Aubl.

Además de las especies dominantes citadas, en este estrato se encuentran:

Calophyllum brasiliense Camb. var. rekoi Standl.

Cymbopetalum baillonii R. E. Fr. 


\section{Lonchocarpus cruentus Lundell \\ Pithecellobium arboreum (L.) Urban \\ Pterocarpus belizensis Standl. \\ Trichilia tomentosa HBK. \\ Virola multiflora (Stadl.) A. C. Smith}

El estrato arbóreo medio de 7 a $12 \mathrm{~m}$. de alto, constituido por:

Guarea glabra Vahl.

Hampea rovirosae Standl.

Lonchocarpus santarosanus Donn. Sm.

Malmea depressa (Baill.) R. E. Fries

Mortoniodendron sp.

Psychotria chiapensis Standl.

Trichilia glabra L.

El estrato arbóreo bajo de 1.8 a 4 m. de alto, formado por:

Enallagma sp.

Erythrina americana L.

Trichilia breviflora Blake et Standl.

Entre los bejucos más frecuentes están:

Connarus schultesii Standl.

Hippocratea celastroides HBK.

Paullinia pinnata L.

Smilax domingensis Will.

\section{Fisiografía}

La Laguna del Majahual es una típica laguna cráter de forma casi circular, con diámetro de unos $500 \mathrm{~m}$. de longitud y en su centro una profundidad de 37 m. (según el Sr. Juan Ruiz López habitante de la zona).

Está localizada $18^{\circ} 40^{\prime} \mathrm{N}$., $95^{\circ} 19^{\prime} \mathrm{P}$., a sólo $5 \mathrm{~km}$. al sur de la línea del litoral, del lugar denominado Puntillas de la costa del Golfo de México; su altitud es de $110 \mathrm{~m}$. sobre el nivel del mar.

Alimentan a la Laguna dos riachuelos en su orilla sur y tiene su salida en el Río Michapan en el margen NO de la Laguna. Los taludes del cráter son los que sustentan vegetación original, el talud sur es el más alto, con unos 30 a $35 \mathrm{~m}$., el norte es el más bajo de 12 a $15 \mathrm{~m}$. y el oriental y occidental de 20 a $25 \mathrm{~m}$. de alto. El área circundante a la Laguna está casi totalmente alterada por siembras de caña de azúcar. 


\section{Clima}

La estación más próxima es la de Angel R. Cabada, a $18 \mathrm{~km}$. al SO de la Laguna del Majahual. Su fórmula climática es Am (e) de acuerdo con la clasificación de Koeppen modificado por García (1964), con 2115.7 mm. de lluvia anual y con una temperatura media anual de $26.0^{\circ}$ C. Siendo cuatro los meses secos, de febrero a mayo, julio es el mes más lluvioso, con una definida sequía intraestival durante agosto. La oscilación térmica anual es amplia, siendo el clima de tipo extremoso. Esto se debe a que durante el invierno la temperatura se abate un poco más que en otras zonas por la influencia de los nortes, además de que durante mayo, el mes más cálido, la temperatura se eleva por la baja humedad atmosférica.

Los nortes se dejan sentir en forma intensa en el área de la Laguna motivados por su proximidad al océano, así como, aparentemente se produce un embalse eólico por el encajonamiento que forma la vertiente occidental de la Sierra de Los Tuxtlas. En el mapa de oscilación térmica anual (García 1968) se aprecia una lengüeta de clima extremoso que bordea al margen occidental de la Sierra de los Tuxtlas, lengüeta que interpreta García como motivada por los nortes (ver mapa II).

La oscilación de la temperatura mensual está estrechamente correlacionada con el régimen de lluvias, especialmente durante los cuatro meses secos, en los que la oscilación llega a su máximo, así como ésta aumenta durante la canícula; en el período de meses húmedos la correlación es menor, dependiendo más bien de la estación del año.

El clima Am es un clima propio para la selva perennifolia (ver Miranda \& Hernández, 1963); sin embargo, en los alrededores de la Laguna del Majahual la vegetación es caducifolia en la orilla norte y sur de la Laguna; no ocurre lo mismo en el talud oriental y occidental. Algo semejante pasa en las pocas elevaciones que se presentan de la Laguna hacia el mar. En dichas elevaciones la cara sur es perennifolia y las otras son caducifolias. Es decir la cara y taludes protegidos de los fuertes vientos del norte retienen sus hojas debido a que el régimen de lluvias es lo suficientemente alto para permitírselos. Así, a finales del período de los nortes, es decir febrero, la vegetación adquiere este aspecto tan peculiar.

\section{Discusión y conclusiones}

Creemos que las aves son las responsables de la concentración de especies con características de la Teoría del Durian (Corner, op. cit., 1953-54) en la Laguna del Majahual. 
La avifauna de Los Tuxtlas es responsable de esta concentración, ya que, de las aves observadas sólo dos especies migratorias son frugívoras, lo que concuerda con el análisis de dispersión endozoica hecho por Steenis (1935) para el origen de la flora de las montañas de Malasia.

Las aves migratorias no son en general frugívoras, y la digestión en las aves es bastante rápida; Steenis (op. cit.) concluye que la dispersión endozoica es significativa dentro de distancias cortas, quizá solamente del orden de decenas de kilómetros; compartimos parcialmente esta idea, ya que pensamos que la avifauna de Los Tuxtlas seleccionó a las especies deseadas y las "sembró" en los taludes de la Laguna del Majahual. Sin embargo, la dispersión endozoica es efectiva a largo plazo, confiriendo a las especies vegetales una distribución continua.

En este punto entendemos quiénes son los responsables de la concentración de dichas plantas; el porqué es un problema de comportamiento animal. Existen muy pocos datos al respecto, pero creemos que los "nortes" obligan a las aves a refugiarse en el microhabitat protegido de los taludes perennifolios oriental y occidental de la Laguna. Es difíicl probar que la Laguna sea un refugio, ya que, en los días de "nortes" las aves permanecen sin hacer grandes movimientos y la lluvia y vientos no permiten que sean escuchadas con claridad (comunicación personal del Dr. A. R. Phillips). Sin embargo, en las primeras horas de la mañana (de 6 a 8 a. m.) las aves muestran una gran actividad trófica, lo que ocurre aún durante los "nortes" ya que como ha hecho ver Allee (1926) para Panamá, la máxima velocidad de los vientos ocurre durante las más altas temperaturas del día, habiendo mucho menos viento en las horas más frías de la mañana.

La caducifoliedad de la vegetación circundante a la Laguna del Majahual va acompañada de la falta de frutos, lo que obliga a las aves a ir a lugares perennifolios. Aún dentro de los taludes de la Laguna se presenta el mismo fenónómeno dependiendo de la exposición, como ya se dijo.

En un principio los taludes oriental y occidental de la Laguna debieron ser refugios contra los fuertes vientos de los nortes, pero paulatinamente las aves debieron haber ido sembrando especies vegetales de las que se alimentan, al usar los árboles originales como perchero, de tal forma que posteriormente además de la protección contra la acción eólica la Laguna les ofreció alimento durante esta época desfavorable. Seguidamente las mismas aves y quizá también los roedores debieron dispersar a las especies vegetales deseadas a los otros dos taludes con vegetación caducifolia, estableciéndose así la misma asociación vegetal para los cuatro taludes de la Laguna.

Es bastante sugestivo el hecho de que especies con semillas relativamente 
pequeñas con definida dispersión endozóica por una gran diversidad de aves como son: Bursera simaruba, especies de Trichilia, Paullinia pinnata, diversas especies de Psychotria, Miconia trinervia* (citada por Land, 1963) y Conostegia aff. icosandra** (citada por Willis, 1966) sean especies de distribución sumamente amplia desde Sudamérica hasta extensas zonas cálidas de nuestro país. En cambio, especies con semillas grandes como Connarus y Swartzia de aparente dispersión endozóica por contadas especies de aves, tienen una distribución restringida y son bastante escasas en las áreas en las que se les encuentra.

Es evidente que las aves juegan un papel fundamental en la dispersión de ciertas especies en las zonas tropicales así como en la germinación de las simientes, en unos casos como escarificadores químicos (como seguramente ocurre en Bursera simaruba, Trichilia spp. y Psychotria spp.), o bien como ha hecho ver Janzen (op. cit.) en Acacia cornigera la supervivencia de las semillas es debida casi en su totalidad a las aves (como Psilorhinus morio), las cuales excretan a las semillas viables lejos de la planta madre que está plagada por brúquidos, insectos que destruyen las semillas que no son retiradas a tiempo por las aves.

Los resultados y conclusiones son preliminares, por lo que se sugiere abordar en un futuro los siguientes aspectos:

a. Observaciones prolongadas de aves frugívoras en la Laguna del Majahual, abarcando un ciclo anual, en su mayor amplitud, o al menos un período de nortes.

b. Localización de otra laguna comparahle a la del Majahual y correlacionar datos.

c. Hacer estudios sobre el comportamiento de las aves, en especial sus movimientos dentro de la región que habitan, tratando de establecer si tienen rutas de alimentación relacionadas con los diferentes eventos climáticos (nortes, estación seca, estación de lluvias. canícula, etc.). Así como si las especies siquen rutas determinadas cada día; si fuera así, su amplitud y recorrido.

d. Registrar datos microclimáticos de los refugios (talud oriental y occidental) y compararlos con los taludes desprotegidos (norte y sur). Haciendo espe cial énfasis en: relocidad de los vientos, humedad relativa y temperatura.

La falta de información en este campo de dispersión endozóica es muy gran. de, lo que dificulta.la confrontación de las observaciones hechas, pero a la vez abre grandes posibilidades en la investigación de la relación animal-planta en los climas cálido-húmedos.

* Especie de Melastomatacea de la cual se alimentan 20 especies de aves en Guatemala.

** Especie de Melastomatacea de la cual se alimentan 28 especies de aves en Colombia. 


\section{LITERATURA}

AlLeE, W. C.

1926 Measurement of Environmental Factors in the Tropical Rain Forest of Panamá. Ecology 7(3) :273-301.

Andrle, R. F.

1967 Birds of the Sierra de Tuxtla in Veracruz Mexico. The Wilson Bull. 79(2) :163-187. Corner, E. J. H.

1949 The Durian Thcory or the Origin of the Modern Tree. Ann. Bot. n. s. 13(52): 367-414.

Corner, E. J. H.

1953-54 The Durian Theory extended, Parts I, II, III. Phytomorphology 3:465-76; 4:152$65,263-74$.

Diamond, J. M. \& J. W. Terborgh

1967 Observations on Bird Distribution and Feeding Assemblages Along the Rio Callaria, Department of Loreto, Perú. The Wilson Bull. 79(3) :273-282.

Friedlaender, I., u. R. A. Sonder

1924 Über das Vulkangebiet von San Martín Tuxtla in Mexiko. Zeltschr. für Vulkanol. $7: 162-87$.

García, Enriqueia

1964 Modificaciones al sistema de clasificación climática de Koeppen. México, D. F.

1968 Los climas del Estado de Veracruz, (Según el sistema modificado por la autora ${ }^{1}$ ). Conferencia sustentada en la Sociedad Botánica de México el 4 de abril. Inédito.

Hespenheide, H. A.

1966 The Selection of Seed Size by Finches. The Wilson Bull. 78(2) :191-97.

JANZEN, D. H.

Seed Eaters, and Seed Size, Number, Toxicity and Dispersal. Evolution, en prensa.

LAND, H. C.

1963 A Tropical Feeding Tree. The Wilson Bull. 75:199-200.

Miranda, F. \& E. Hernández X.

1963 Los tipos de vegetación de México y su clasificación. Bol. Soc. Bot. Méx. 28:29-179.

Sarukhan, K. J.

1963 Palo Mulato. Seminarios, E. N. A. Centro de Genética. Colegio de Postgraduados, Chapingo. México.

Steenis, C. G. J. van

1935 On the Origin of the Malaysian Mountain Flora.

Part 2. Altitudinal Zones, General Considerations and Renewed Statement of the Problem.

Buitenzorg Jard. Bet. Bull. Ser. 3, 13:289-417.

Wetmore, A.

1943 The Birds of Southern Veracruz, Mexico. Proc. U. S. Natl. Mus. 93:215-340.

Willis E. O.

1966 Competitive exclusion and birds at fruiting trees in Western Colombia. The Auk 83 (3) $: 479-480$. 

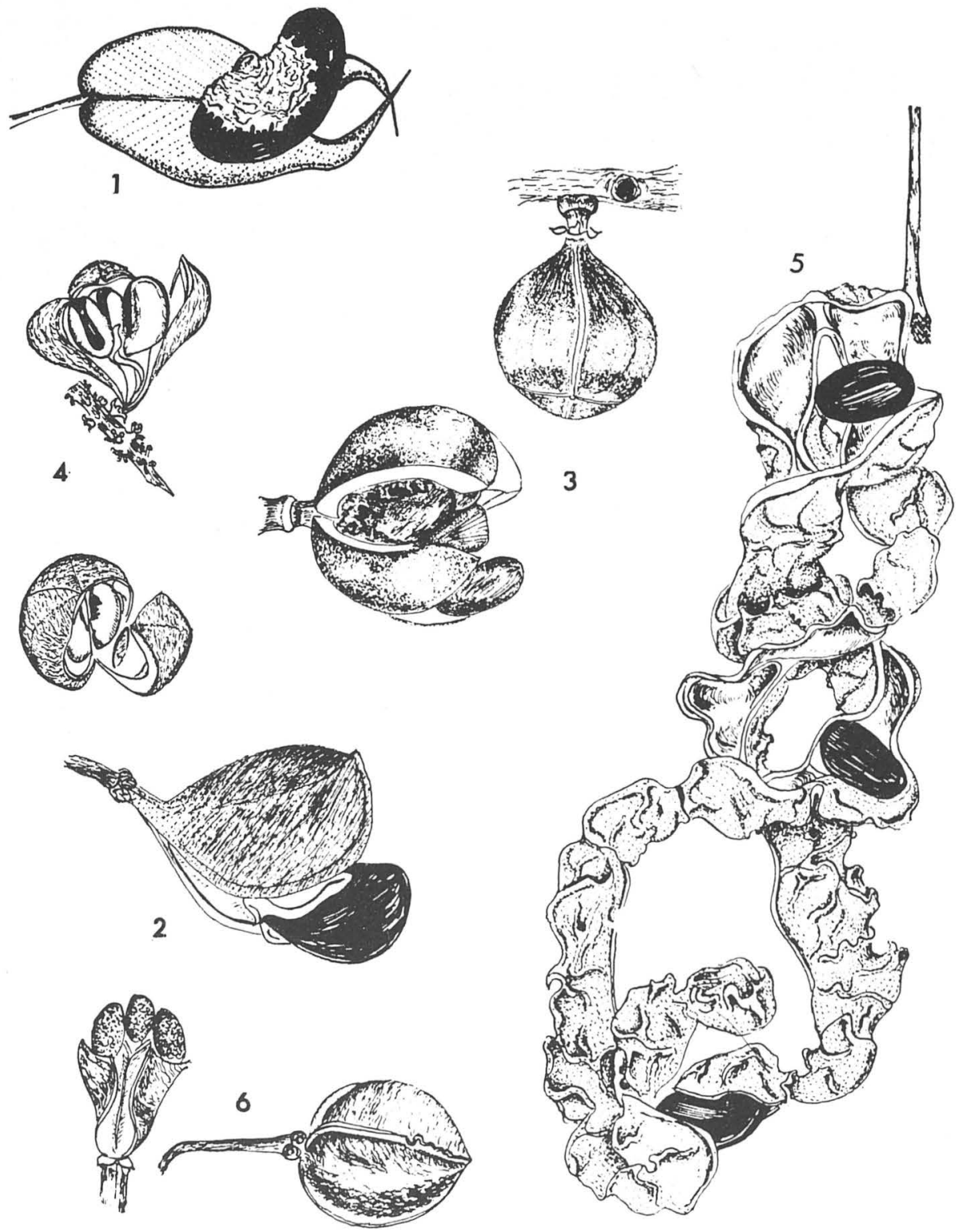

Fig. 1. Swartzia guatemalensis (Donn. Sm.) Pittier, Col. M. Sousa 3339 (MEXU). Escala 1: 1. Fig. 2. Connarus schultesii Standl. Col. M. Sousa 3338 (MEXU). Escala 1: 1. Fig. 3. Guarea glabra Vahl Col. M. Sousa 3336 (MEXU). Escala 1: 1. Fig. 4. Paullinia pinnata L. Col. M. Sousa 3337 (MEXU) Escala 1: 1. Fig. 5. Pithecellobium arboreum (L.) Urban Col. M. Souza 3335 (MEXU) Escala 1: 1. Fig. 6. Mortoniodendron sp. Col. M. Sousa 3340 (MEXU). Escala 1: 1. 


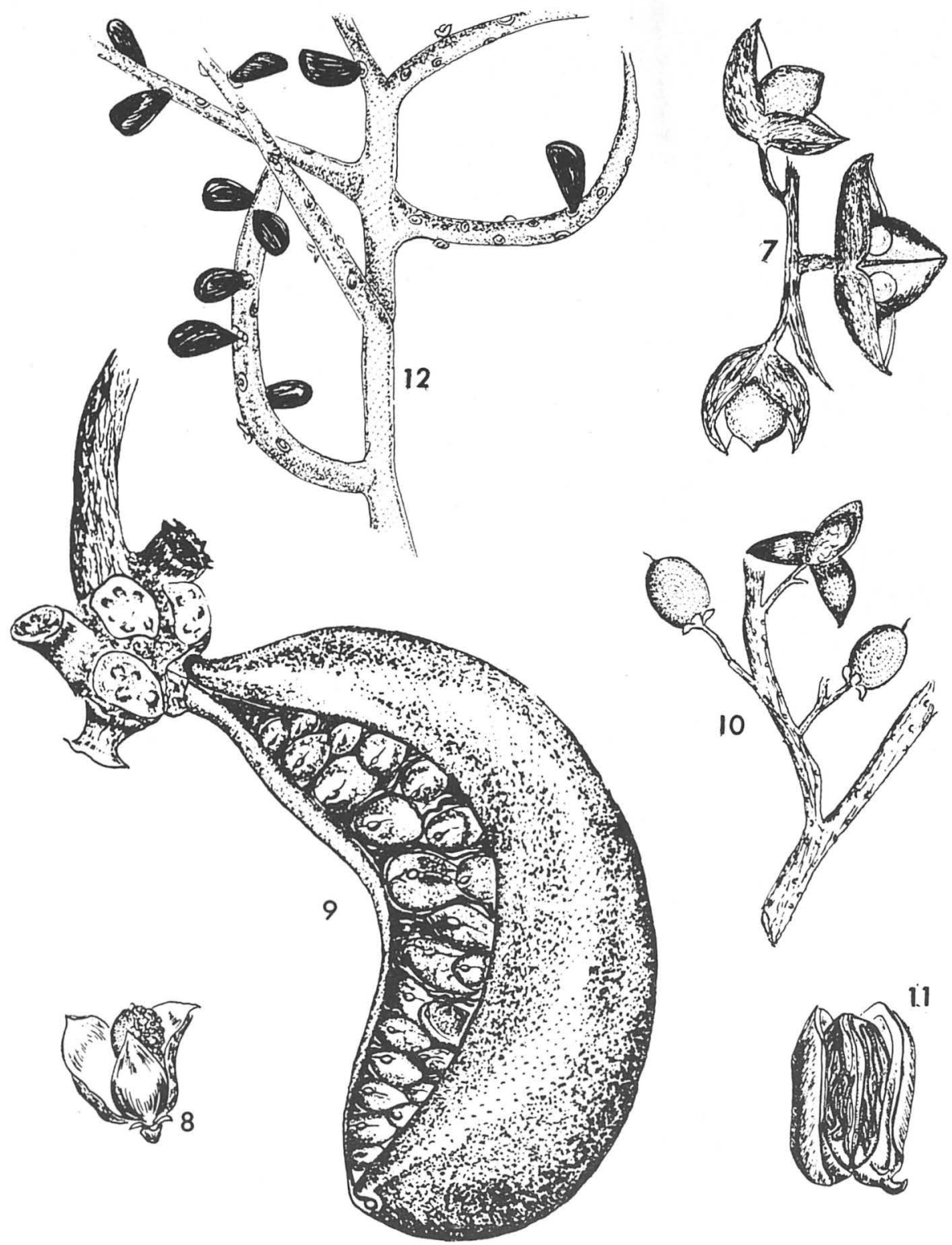

Fig. 7. Trichilia tomentosa HBK. Col. M. Sousa 3341 (MEXU). Escala 1: 1. Fig. 8. Trichilia breviflora Blake et Standl. Col. M. Sousa 3362 (MEXU). Escala 1: 1. Fig. 9. Cymbopetalum baillonii R. E. Fr. Col. M. Sousa 3382 (MEXU). Escala 1. 1: Fig. 10 Casearia nitida (L.) Jacq. Col. M. Sousa 3359 (MEXU). Escala 1: 1. Fig. 11. Virola multiflora (Standl.) A. C. Smith Col. M. Sousa 3361 (MEXU). Escala 1: 1. Fig. 12 Chamaedorea tepejilote Leibm. Col. M Sousa 3334 (MEXU) Escala 1: 1. 

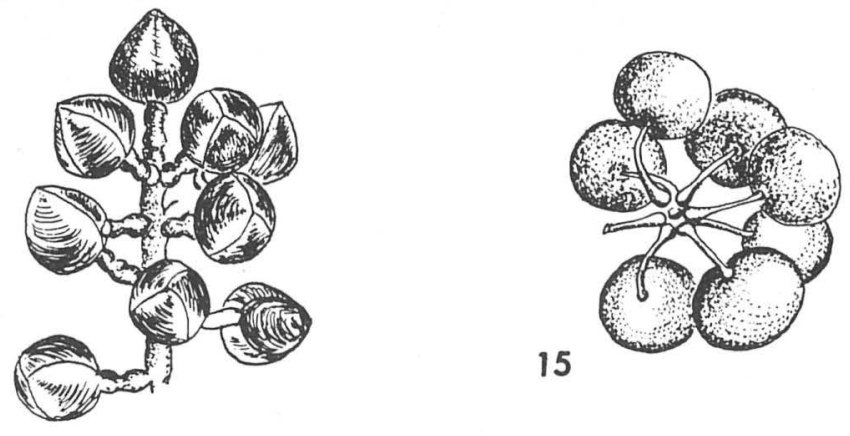

13
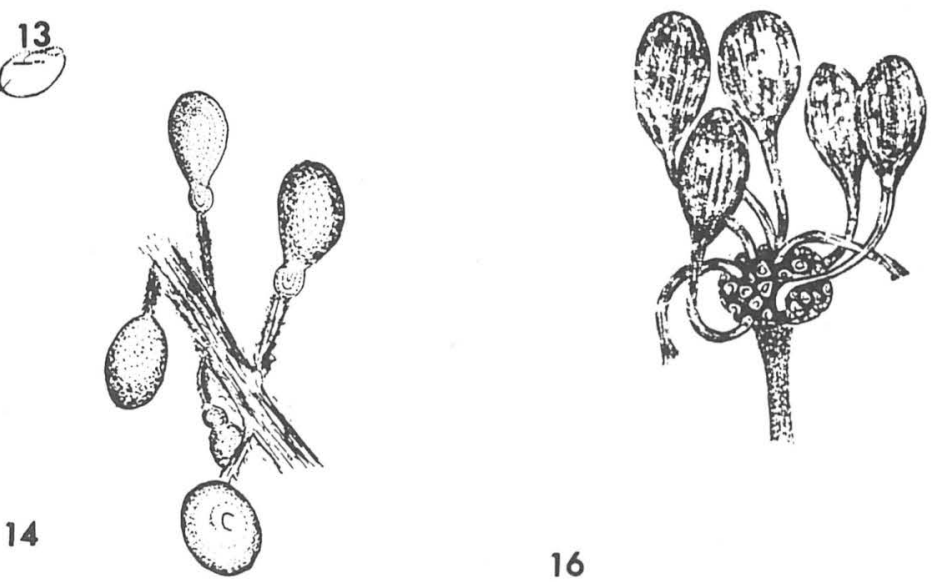

Fig. 13. Bursera simaruba (L.) Sarg. Col, M. Sousa 3390 (MEXU). Escala 1: 1. Fig. 14. Psychotria uliginosa Standl. Col. M. Sousa 3358 (MEXU). Escala 1: 1. Fig. 15 Smilax domingensis Willd. Col. M. Sousa 3411 (MEXU). Escala 1: 1. Fig. 16. Malmea depressa (Baill.) R. E. Fries. Col. M. Sousa 3391 (MEXU). Escala 1: 1. 


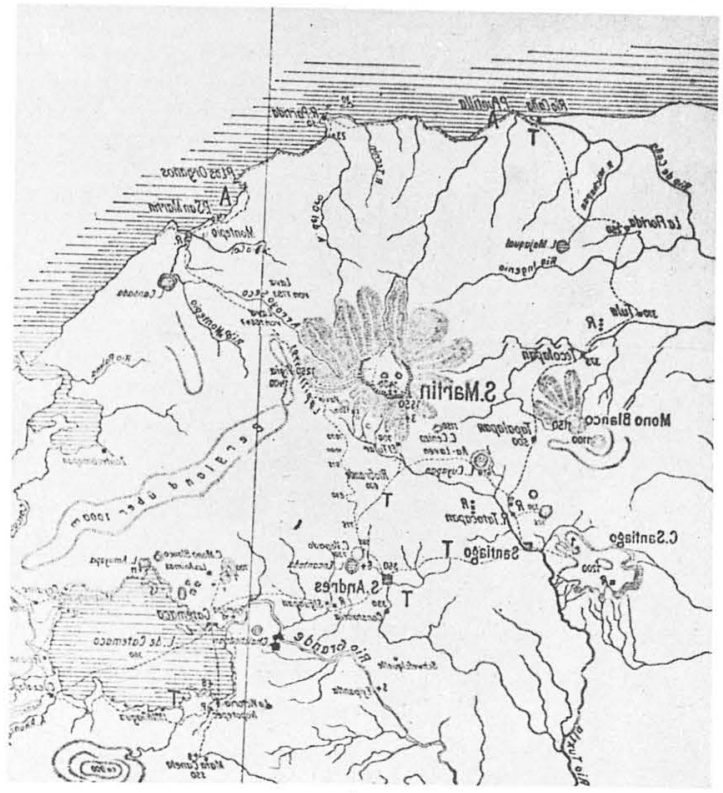

Mapa I Noreste de la región de los Tuxtlas, Ver. (Tomado de Friedlaender \& Sonder, 1924).

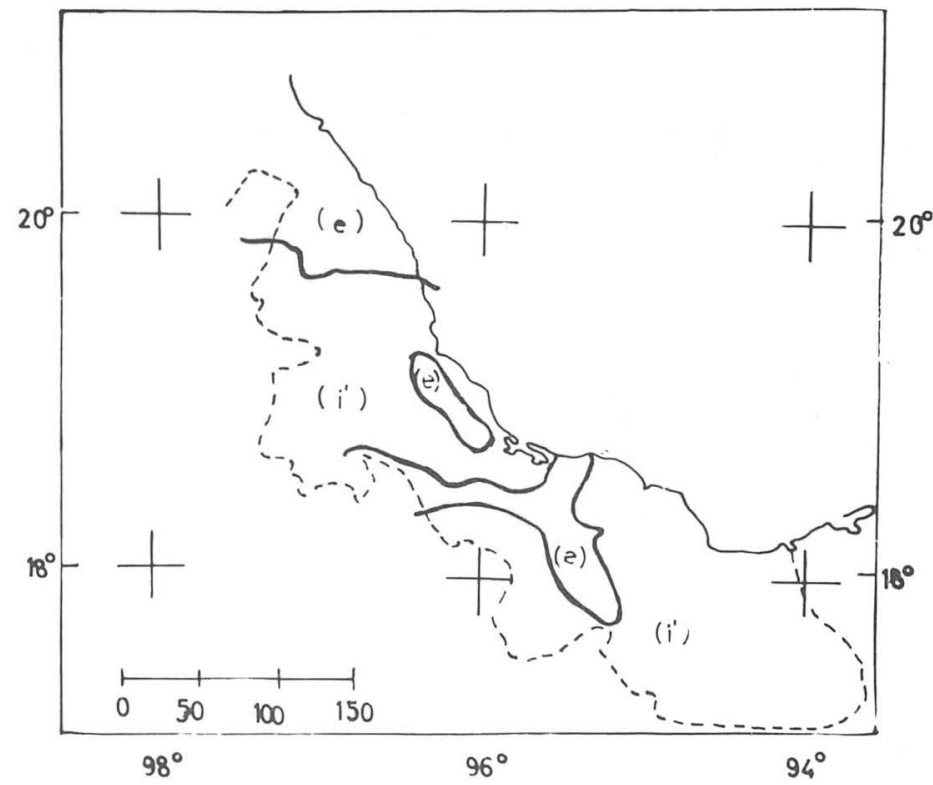

Mapa II Oscilación térmica anual, del sur del Edo. de Veracruz. 'Tomada de García). Clave: (i') climas de poca oscilación (entre $5^{\circ}$ y 7 ํㅡ). (e) climas extremosos (con una

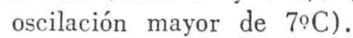


ANGEL R. CABADA AM( \&) (10 años)

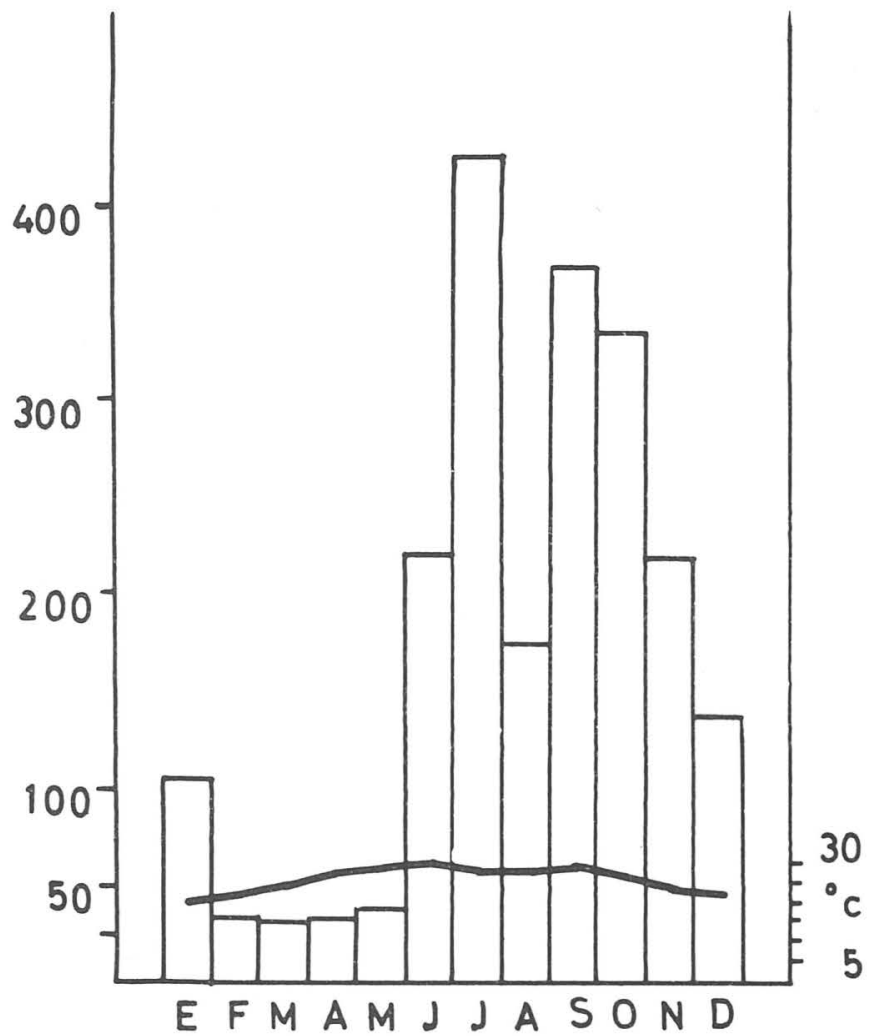

PROMEDIO DE TEMPERATURAS

MAXIMAS Y MINIMAS

(10 años)

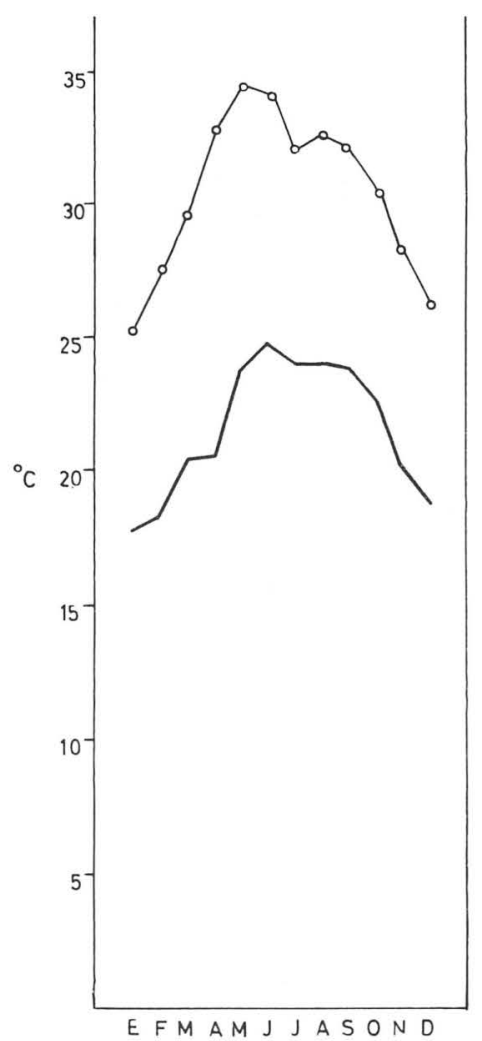

OSCILACION DE

TEMPERAIURA

(10 años)

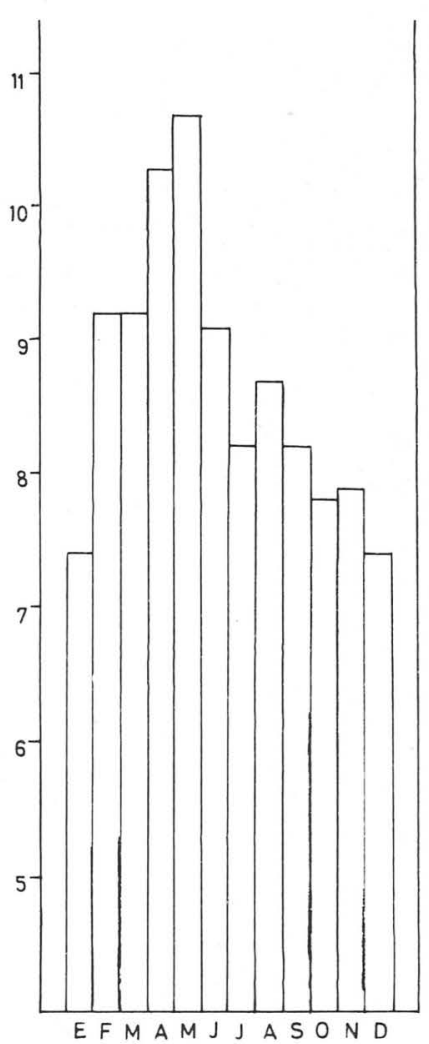

